\title{
Modelo de entrenamiento en toma de decisiones relacionadas con gestión de producción y operaciones de un sistema de fabricación de bioetanol
}

\section{Model training decisions relating to production and operations management system of making bioetanol}

\author{
Jhonathan Mauricio Vargas-Barbosa \\ M. Sc (c) Ingeniería Industrial, \\ Universidad Nacional de Colombia \\ Manizales, Colombia \\ jmvargasba@unal.edu.co
}

\author{
Jaime Alberto Giraldo-García \\ Ph. D. Ingeniería-automatización Industrial, \\ Universidad Nacional de Colombia \\ Manizales, Colombia \\ jaiagiraldog@unal.edu.co
}

\begin{abstract}
Resumen- El objetivo de este trabajo de investigación fue desarrollar un modelo de decisión para que estudiantes de Ingeniería Química se entrenen en la toma de decisiones relacionadas con la Gestión de la Producción y las Operaciones (GPO). El modelo está soportado en simulación discreta, y mediante este los usuarios pueden plantear diferentes escenarios de operación de un proceso típico abordado por la ingeniería química, como lo es la producción de bioetanol a partir de caña de azúcar, y evaluar cómo estos escenarios afectan el nivel de producción. Se espera que el modelo sea utilizado en el entrenamiento de futuros ingenieros químicos en toma de decisiones sobre GPO, para facilitar la experimentación con un sistema real mediante su simulación. Se concluye la importancia del uso de la simulación en los procesos formativos, mediante modelos y herramientas construidos entre docentes y estudiantes, que permitan romper con las barreras espaciales, temporales y de costo al experimentar con sistemas reales y así fomentar la formación en ambientes virtuales de aprendizaje.

Palabras clave- Gestión de la producción y operaciones, modelo de decisión, proceso enseñanza-aprendizaje, simulación, sistema de producción de bioetanol.
\end{abstract}

Abstract- The objective of this research was to develop a decision model for chemical engineering students are trained in making decisions related to the Management of Production and Operations (GPO). The model is supported in discrete simulation and through this, users can pose different scenarios of operation of a typical process addressed by the chemical engineering, such as the production of bioethanol from sugarcane and evaluate how these scenarios affect production level. Model is expected to be used in the training of future chemical engineers in making decisions about GPO, facilitating experimentation with a real system by simulation. The importance of the use of simulation in training processes, using models and tools built between teachers and students, allowing break the space, time and cost to experiment with real systems and barriers and promote training in virtual environments is concluded learning

Keywords- Production Management and Operations, Model Decision Process Teaching / Learning, Simulation, Bioethanol Production System.

\section{INTRODUCCIÓN}

Este trabajo reporta el desarrollo de un modelo de decisión de soporte a un sistema de producción de bioetanol (a partir de caña de azúcar) apoyado en simulación, mediante el cual se controlan variables de decisión relacionadas con la GPO, que afectan el desempeño del sistema, en términos del volumen de producción de bioetanol (en litros) en un periodo de tiempo determinado. El objetivo central es entrenar a estudiantes de ingeniería química en la toma de decisiones en temas relacionados con GPO, empleando el modelamiento de sistemas comúnmente abordados en los currículos de ingeniería química. La idea se gesta a partir de indagaciones preliminares que revelan que alrededor del $90 \%$ de los estudiantes de esta carrera, en nuestra sede universitaria, encuentran dificultades en la enseñanza-aprendizaje de este tema.

En la actualidad la generación de alternativas energéticas distintas a las ya convencionales, obtenidas principalmente de la explotación del petróleo, ha conllevado al uso de materias pri- 
mas naturales en la fabricación de los llamados biocombustibles, entre los cuales se destaca el bioetanol. Para Sánchez et al. [1] la produccion de energías renovables ha surgido a raíz de la necesidad de proteger el medio ambiente, preservar los recursos, tanto renovables como no renovables y maximizar el potencial de uso de productos agrícolas, y en especial, de los subproductos que estos generan al someterlos a distintos procesos agroindustriales, cuya disposición final es un gran problema ambiental.

El proceso para la producción de etanol por vía fermentativa o bioetanol tiene dos etapas fundamentales: la fermentación y la destilación [2]. Vázquez y Dacosta [3] definen la fermentación como una bioreacción que permite degradar azúcares en alcohol y dióxido de carbono. Geankoplis [4] considera la destilación como un método de separación de componentes de una solución líquida, la cual depende de la distribución de los componentes entre una fase de vapor y una fase líquida. En la Fig. 1 se muestra un diagrama simplificado del proceso de producción de bioetanol cuyas etapas fundamentales son la fermentación y la destilación.

Pero además de considerar los aspectos técnicos del proceso, estos sistemas requieren ser gestionados apropiadamente para alcanzar los niveles de producción deseados. La GPO busca continuamente la construcción y sostenibilidad de las capacidades competitivas de la unidad empresarial, lo que implica la comprensión del rol de todas las funciones organizacionales y en especial la de producción como función clave dentro del quehacer empresarial, y su contribución para enfrentar los actuales condicionamientos del entorno [5]. Según la legislacion del Congreso de la República de Colombia [6], se define la profesión de ingeniería química como "la aplicación de los conocimientos y medios de las ciencias físicas, químicas y matemáticas, en el análisis, diseño, dirección, supervisión y control de procesos de transformacion de materias primas en productos terminados", lo cual evidencia una relacion directa entre la GPO y la ingeniería química.

Para entender esta relacion se requiere conocer de manera cuantificada el aspecto operativo del sistema por gestionar. La Fig. 2 muestra un esquema operacional básico de flujos de material de un sistema de producción de bioetanol en par- ticular. A partir de un flujo de caña de $1000 \mathrm{~kg} /$ hora, el cual para su lavado requiere $5000 \mathrm{l} /$ hora de agua para remover las impurezas que podrían contaminar los jugos azucarados en la fermentación. La caña debe pasar por un proceso de molienda en el que se extraen los jugos azucarados y como subproducto se obtiene bagazo, el cual en muchas plantas es utilizado para la generación de energía mediante la combustión. Se estima que por cada kilogramo de caña, el 73\% representa los jugos azucarados. A estos jugos se les debe agregar algunos nutrientes y sustancias químicas para adecuar las condiciones para la fermentación donde los microorganismos digieren los azucares y los transforman en etanol; al producto de la fermentación se le conoce como mosto. Este último contiene etanol y otras sustancias, por tanto, es necesario separar el producto de interés (etanol) de los subproductos contaminantes, por ello se pasa esta mezcla por dos etapas de destilación. La primera separa el etanol del resto de sustancias hasta una concentración alrededor del 95\% en volumen, el cual representa el punto azeótropo (máxima concentración de etanol en condiciones normales). Luego, se pasa a la segunda etapa de destilación donde se modifican las condiciones de temperatura y presión para obtener un producto $100 \%$ puro. Como subproducto de la destilación se obtienen vinazas las cuales contienen gran cantidad de materia orgánica que debe ser tratada antes de ser vertida en los afluentes. Se estima que por cada litro de etanol producido se obtienen alrededor de 9.7 litros de vinazas, las cuales representan un problema ambiental al no ser tratadas como corresponden.

Estos sistemas en el mundo real consideran múltiples maquinas/equipos, áreas de almacenamiento, recursos de transporte, diversos flujos de información y material, procedimientos y reglas de control, entre otros. Cuando estos se modelan debe procurarse representar gran parte de su estructura y comportamiento (lo que le da robustez al modelo), si se quieren tener modelos válidos del sistema. Con las herramientas de simulación se pueden desarrollar modelos robustos que se acercan a la realidad estudiada, cuestión que no pasa con otras herramientas de soporte a la toma de decisiones, tales como: la programación lineal o algunos métodos de solución analítica como el cálculo (diferencial e integral) y el álgebra, entre 
Fig. 1. ESQUEMA SIMPLIFICADO DE PRODUCCIÓN DE BIOETANOL A PARTIR DE CAÑA DE AZÚCAR

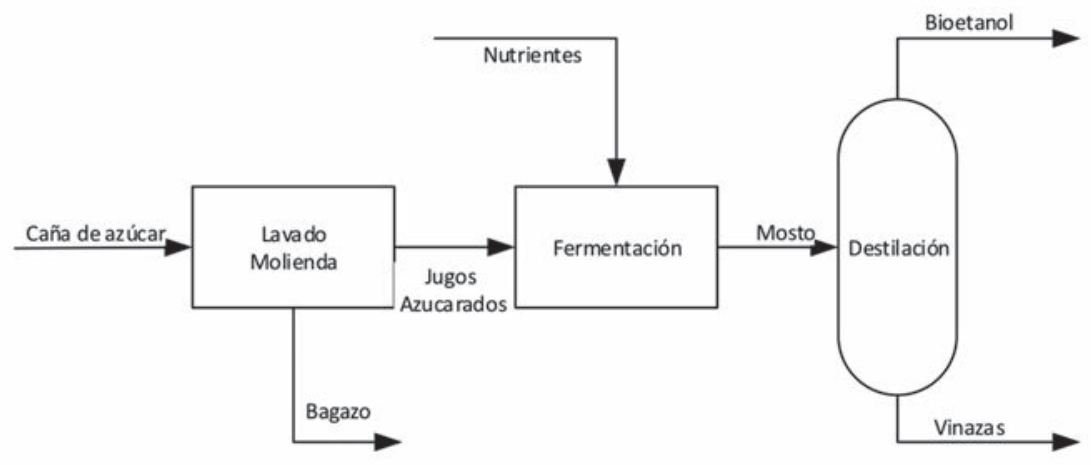

Fuente: autores, a partir de la observacion directa en un sistema real.

Fig. 2. ESQUEMA BÁSICO DE UN SISTEMA DE PRODUCCIÓN DE BIOETANOL

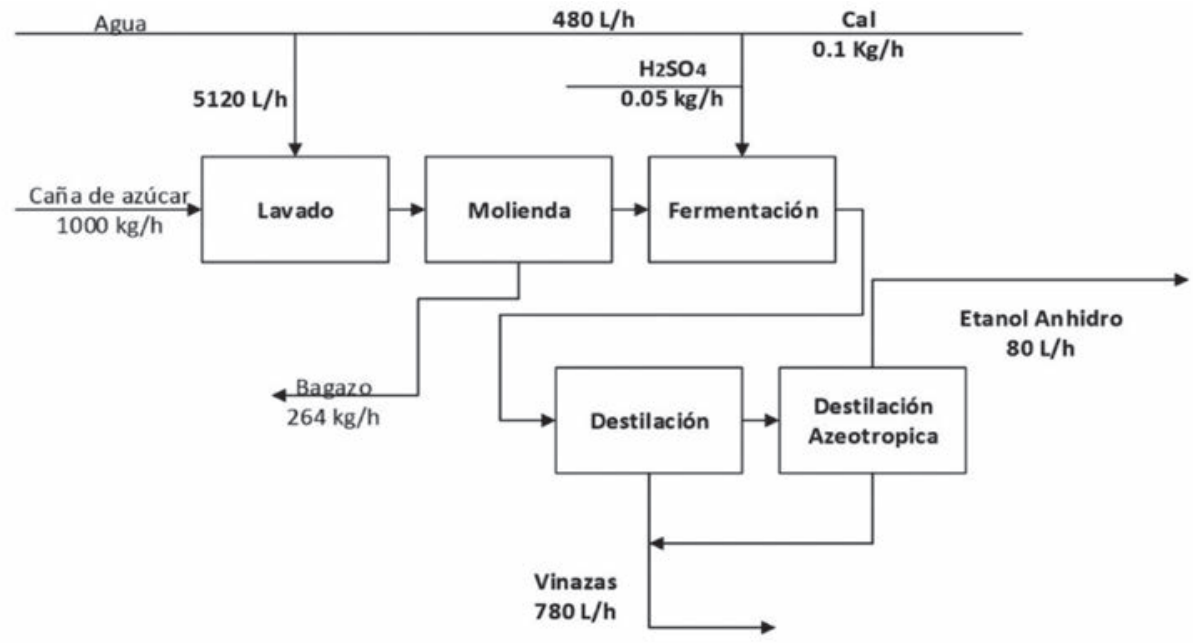

Fuente: autores, a partir de la observacion directa en un sistema real.

otros [7]. El uso de simulación está relacionado con la complejidad de los sistemas estudiados, haciendo intervenir variables controlables y no controlables, con características estocásticas y alta interdependencia entre componentes, mientras que con el uso de otras herramientas matemáticas se deben proponer algunas hipótesis de simplificación, las cuales alejan el sistema estudiado de su realidad.

Dado que el sistema para gestionar presenta comportamiento estocástico y alta interdependencia, Kelton y Law [7], Banks et al. [8] y Shannon [9] están de acuerdo con lo que se ha de considerar el modelamiento de sistemas complejos mediante simulación si:

- No existe una formulación matemática del problema
- Las técnicas analíticas están disponibles, pero los procedimientos matemáticos son tan complejos y difíciles que la simulación proporciona un método más simple de solución.

- Se desea observar el comportamiento simulado del sistema sobre un periodo de tiempo.

- Se requiere la aceleración del tiempo para sistemas que exigen más tiempo para realizarse.

En la literatura se ofrecen muchas metodologías aceptadas para la construcción de modelos y estudios con simulación. Giraldo [10], uno de los autores, en su libro plantea una adaptación a las metodologías propuestas por Banks et al. (2009), Kelton y Law (2007) y Harrell et al. (2004), entre otros. Esta adaptación metodológica se empleó en el desarrollo del modelo de simulación que se describe adelante. 
Finalmente, al hacer un corto sondeo en la literatura especializada sobre simulación de sistemas de producción de bioetanol, se encuentra que autores como [11], [12] y [13] entre muchos otros, han realizado trabajos enfocados a la simulación de procesos de producción de bioetanol por diferentes medios. [11] hace un análisis de las tecnologías existentes para la obtención de etanol a partir de dos materias primas (maíz y caña de azúcar), realiza una simulación y valida los datos con procesos reales. Para finalizar, concluye que la simulación es una herramienta muy importante, ya que reduce costos, minimiza tiempo y permite una experimentación más amplia en los bioprocesos. Aunque [11] expresa las ventajas de la simulación en procesos industriales como la producción de bioetanol, no propone utilizar esta herramienta como ayuda didáctica. En su estudio, [12] utilizó la simulación como herramienta para estimar algunas propiedades fisicoquímicas de las materias primas, obtener resultados del funcionamiento de la planta y estimar los costos energéticos. Aunque este estudio propone un proceso alterno para la obtención de bioetanol, la simulación no es utilizada con fines pedagógicos. [13] en su tratado hace un análisis de la producción de etanol por hidratación de etileno. Como otros autores, [13] utiliza la simulación para obtener resultados del funcionamiento del proceso propuesto, estimar costos y proponer mejoras. Aunque los modelos de simulación propuestos por los autores [11], [12] y [13] entre otros, son muy útiles para el diseño y mejora de procesos, estos no están enfocados como herramientas didácticas que ayuden a los estudiantes al entrenamiento en temas relacionados con la GPO.

Motivados por lo antes expuesto, en este artículo se presenta un modelo de decisión soportado en simulación como herramienta de entrenamiento en GPO, con el fin de que estudiantes de ingeniería química estén preparados en el ámbito laboral al momento de tomar decisiones en su desempeño profesional.

\section{DESARROLLO METODOLÓGICO}

\subsection{Detección de dificultades en los procesos de enseñanza-aprendizaje en GPO}

El aumento de estudiantes y profesionales de ingeniería química que han cursado tanto la Es- pecialización en Dirección de Producción y Operaciones como la Maestría en Ingeniería Industrial (programas ofrecidos por el Departamento de Ingeniería Industrial de nuestra Universidad), muestran la necesidad de abordar temas referentes a la GPO en el programa de Ingeniería Química. Prueba de ello es que para el 2013 se registraron 33 estudiantes en los posgrados referidos, los cuales son ingenieros químicos o estudiantes de Ingeniería Química que asisten a estos posgrados como opción de grado.

Para el estudio de las dificultades en los procesos de enseñanza-aprendizaje de la GPO para ingenieros químicos se proponen 4 categorías de variables causa-efecto, a incluir en los instrumentos de recolección de datos, las cuales exploran los procesos formativos desde diferentes puntos de vista [14].

- Prácticas en los procesos de enseñanzaaprendizaje de GPO TIC.

- Contexto académico en los procesos de enseñanza-aprendizaje.

- Características de las actividades de enseñanza-aprendizaje basadas en TIC.

- Presencia o ausencia de las TIC.

Se aplicaron encuestas a estudiantes y egresados del programa de Ingeniería Química de la Universidad Nacional de Colombia sede Manizales, en la que se encontró que para una muestra preliminar de 26 egresados y 17 estudiantes del programa, en función de las categorías citadas, el $77 \%$ de los estudiantes encuestados consideran necesario el uso de las TIC en los procesos de enseñanza-aprendizaje y específicamente en temas referentes a la GPO y enfocando estos procesos formativos al uso de casos de estudio soportados con TIC. En el instrumento aplicado a los egresados, este grupo resalta la falencia que encontraron en sus procesos formativos, donde las TIC no hicieron parte fundamental en el aprendizaje de la GPO. El $77 \%$ de los encuestados consideran que las TIC en su pregrado no aportaron a la preparación y desempeño en temas referentes a la GPO. También, se encuentra que el $85 \%$ de los egresados encuestados ha considerado profundizar en temas referentes a la GPO, ya sea por interés propio o porque en su campo laboral han encontrado la necesidad de profundizar en dichos temas. Igualmente, el 
$100 \%$ de los egresados consideran importante el uso de las TIC como soporte en sus procesos formativos, de los cuales el $62 \%$ considera que las herramientas y temas vistos no están enfocados al contexto colombiano. Por otra parte, todos los estudiantes encuestados consideran que el uso de las TIC aporta a los procesos formativos. También en diálogos sostenidos con egresados del programa se ha evidenciado la necesidad e importancia de temas como la distribución en planta, localización, planeación y programación de la producción entre otros temas, para su desarrollo profesional.

Tanto los egresados como los estudiantes destacan entre las muchas características de las TIC, el amplio acceso a la información, la no existencia de barreras espaciales para acceder al proceso formativo, la interacción virtual entre diferentes sujetos involucrados en los procesos de enseñanza-aprendizaje y la facilidad para acceder a la información desde cualquier lugar y en cualquier momento. Ahora, desde la enseñanza, los docentes del programa de Ingeniería Química recalcan también la importancia de la GPO en el ámbito profesional, considerando importante abordar los procesos de enseñanza-aprendizaje soportados en TIC y contextualizando el conocimiento en el ámbito nacional y regional (para ver los resultados de las encuestas, visitar el sitio http://modelogpoeniq.wordpress.com/).

\section{2 Conceptualización del modelo de decisión}

El planteamiento del modelo de decisión se describe en (1) cuyas variables de decisión $x_{i}$ presentan una relación lógico-matemática con la variable respuesta $y$ descrita por la función $f\left(x_{i}\right)$. Los componentes de entrada/salida del modelo se describen en la Tabla I.

TABLA I.

COMPONENTES ENTRADA-SALIDA DEL MODELO DE DECISIÓN

\begin{tabular}{|c|l|c|}
\hline Variable & \multicolumn{1}{|c|}{ Descripción de la variable } & Unidad de medida \\
\hline$X_{1}$ & Coordenada x zona de almacenamiento de caña & Metros $(\mathrm{m})$ \\
\hline$X_{2}$ & Coordenada y zona de almacenamiento de caña & Metros $(\mathrm{m})$ \\
\hline$X_{3}$ & Coordenada x zona de lavado de caña & Metros $(\mathrm{m})$ \\
\hline$X_{4}$ & Coordenada y zona de lavado de caña & Metros $(\mathrm{m})$ \\
\hline$X_{5}$ & Coordenada x zona de almacenamiento de bagazo & Metros $(\mathrm{m})$ \\
\hline$X_{6}$ & Coordenada y zona de almacenamiento de bagazo & Metros $(\mathrm{m})$ \\
\hline$X_{7}$ & Capacidad molienda & Kilogramos $(\mathrm{Kg})$ \\
\hline$X_{8}$ & Capacidad zona lavado de caña & Kilogramos $(\mathrm{Kg})$ \\
\hline$X_{9}$ & Tiempo de molienda según distribución de probabilidad & Horas $(\mathrm{h})$ \\
\hline$X_{10}$ & Tiempo lavado de caña según distribución de probabilidad $(\mathrm{h})$ \\
\hline$y$ & Variable respuesta, producción de etanol en un periodo de tiempo dado & Litros $(\mathrm{L})$ \\
\hline$f\left(x_{i}\right)$ & Modelo de simulación & \\
\hline
\end{tabular}

Se tiene que las variables $X_{1}$ a la $X_{6}$ son coordenadas en un plano cartesiano y las variables $X_{7}$ a la $X_{10}$ representan parámetros de operación de las zonas de molienda y lavado de caña, cuyos valores pueden ser determinísticos o aleatorios.

\section{RESULTADOS Y DISCUSIÓN}

Para Fredes et al. [15], la simulación al propiciar un ambiente virtual puede ser una herramienta muy útil al momento de situar a los estudiantes en escenarios prácticos, que para el caso lo cons- tituye la toma de decisiones en GPO, ya que pueden acceder a modelos de sistemas productivos reales y modificar algunas condiciones de operación, las cuales en el mundo real no lo pueden hacer, por razones que van desde el costo hasta el riesgo de ser sancionados ante pobres decisiones. En la misma vía, López y Saiegg [16] señalan que se puede utilizar la simulación de eventos discretos en los procesos de aprendizaje como una herramienta de experimentación, mejorando la forma en que los alumnos aprenden, permitiendo a estos trabajar con sistemas complejos y que 
difícilmente puedan ser estudiados de forma directa.

Consecuente con lo antes expuesto, se desarrolló un modelo de decisión basado en simulación discreta (ver su representación gráfica en la Fig. 3 y conceptual en la Fig. 4) como herramienta de soporte al proceso enseñanza-aprendizaje de la GPO empleando el software de simulación Flexsim. El empleo de este software obedece a que es un programa de simulación que ha tenido una constante y rápida evolución (una nueva versión en promedio cada 2 años), además de permitir el modelamiento discretofluido de forma completamente gráfica. Mediante el modelo de simulación desarrollado se permite al usuario final ingresar los valores de las variables de decisión $X_{i}$, evaluar cómo se comporta el sistema y cómo estas variables de decisión afectan la producción de bioetanol. Los valores de las variables de decisión son ingresadas empleando la interfaz gráfica de usuario mostrada en la Fig. 5.

Fig. 3. REPRESENTACIÓN GRÁFICA DEL MODELO DE SIMULACIÓN

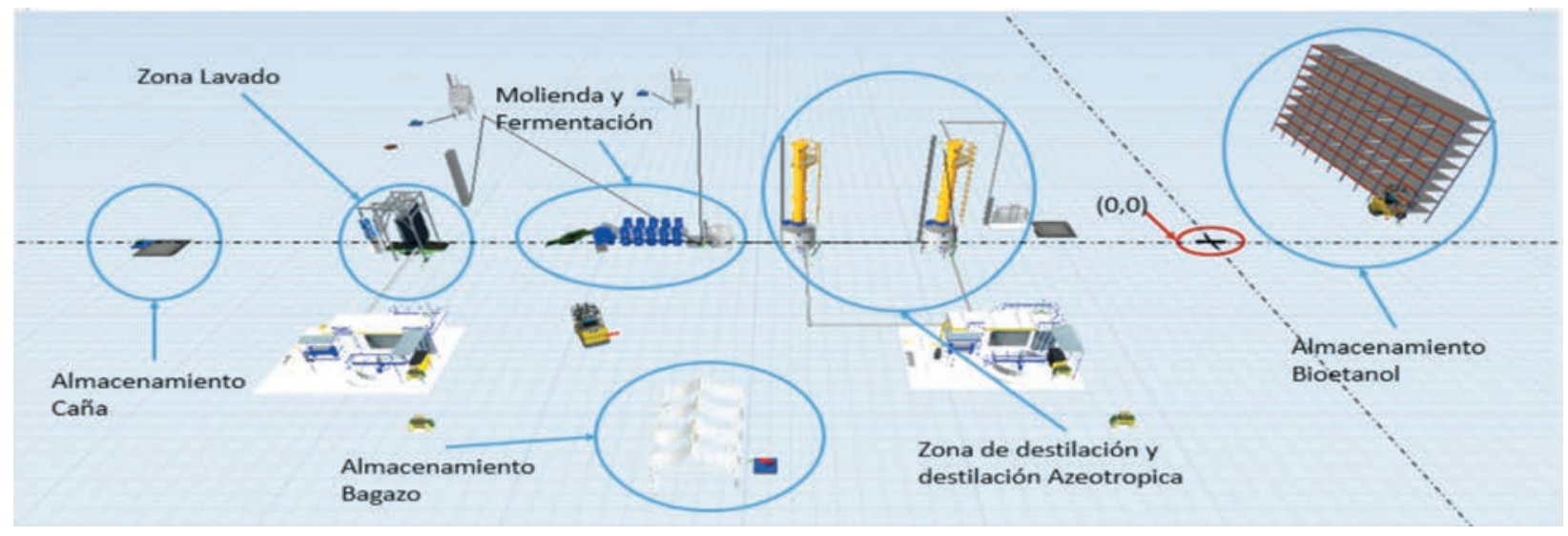

Fuente: autores.

Fig. 4. REPRESENTACIÓN CONCEPTUAL DEL MODELO DE SIMULACIÓN

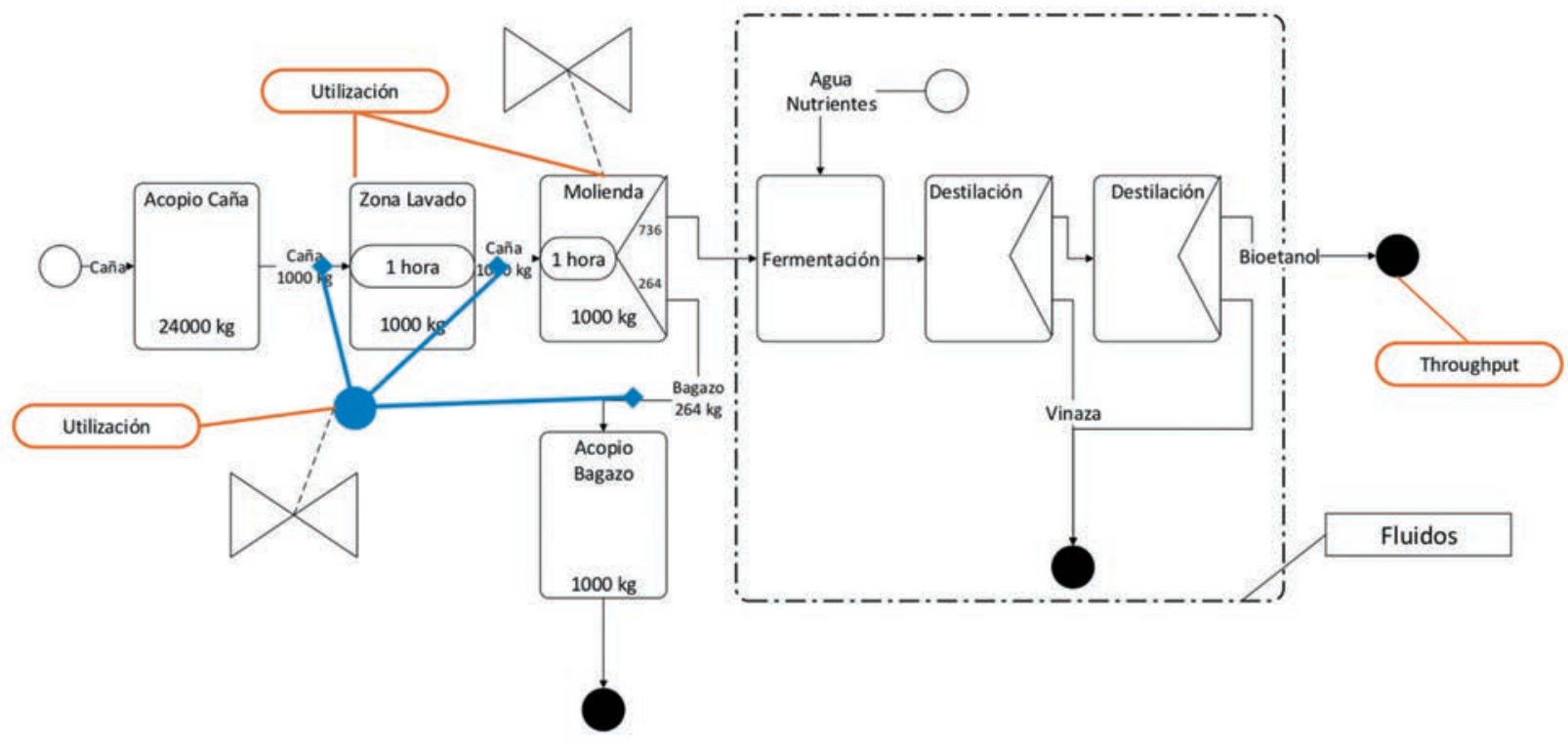

Fuente: autores a partir de simbología propuesta por Flexsim Inc. 
Fig. 5. INTERFAZ GRÁFICA DE INTERACCIÓN USUARIO Y MODELO DE SIMULACIÓN

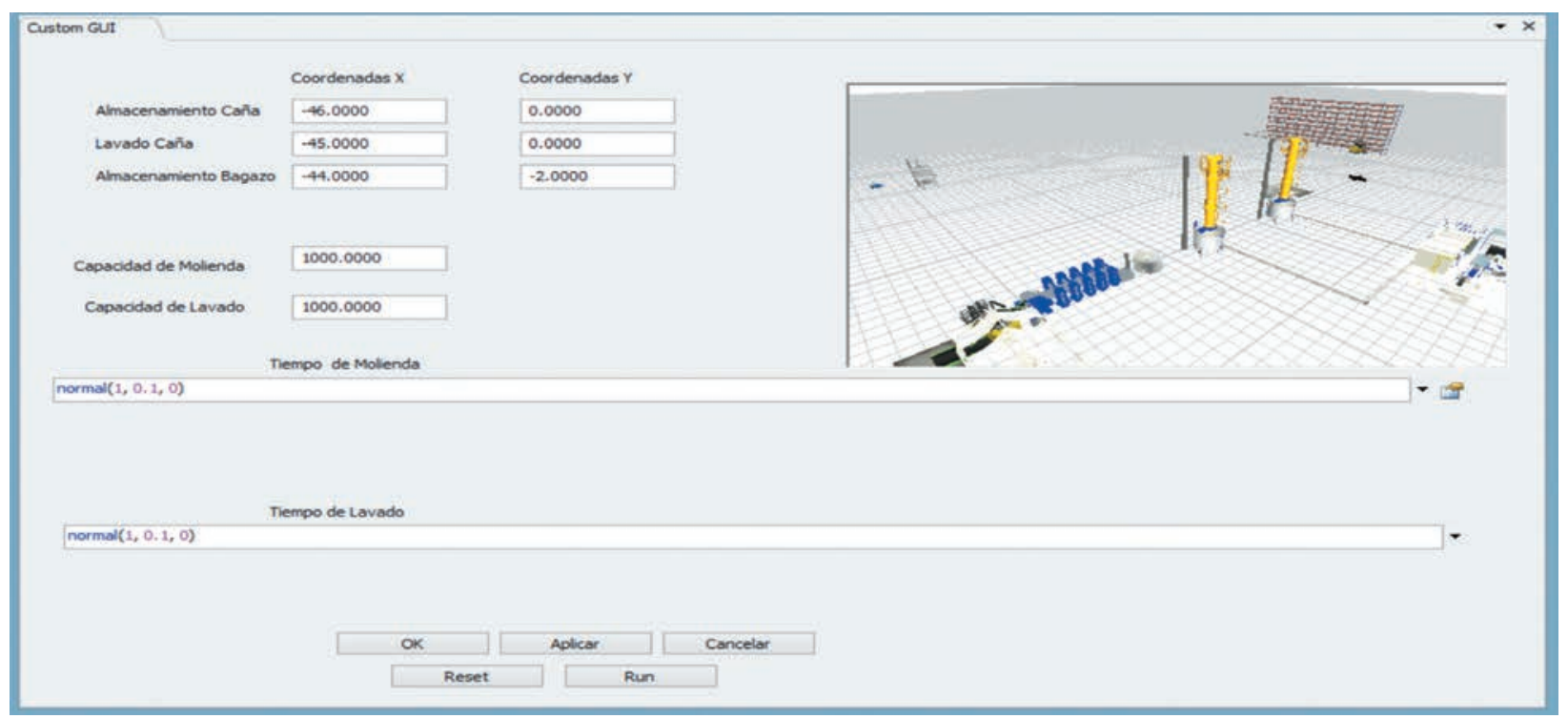

Fuente: autores.

Parte de la robustez del modelo de simulación desarrollado se refleja en el modelamiento de algunas variaciones no controlables como las paradas de los transportadores de caña, las cuales presentan un carácter aleatorio tanto por frecuencia de ocurrencia como duración. También se modelan ocurrencias como paradas totales de la planta para mantenimiento, estas paradas tienen una mayor duración y afectan la producción total de bioetanol. El modelo se desarrolló con estas condiciones, pues se busca representar fielmente el proceso productivo real. Los lectores que estén interesados en conocer y descargar el modelo de simulación desarrollado lo pueden hacer del sitio: http://modelogpoeniq.wordpress.com/

Con fines de experimentación, se utiliza una longitud de corrida de 720 horas (1 mes), pudiendo el usuario modificar esta longitud de corrida según sus propios criterios. En pruebas preliminares se evidencia un periodo de calentamiento de 100 horas donde el sistema comienza a presentar una variación estable en los resultados. Se realizan 5 réplicas por escenario planteado para una simulación piloto, esto con el fin de conocer la variabilidad de los resultados del modelo. Con los valores recolectados se puede proponer un número de réplicas según el nivel de confianza con el que se diseñen cada una de las experimentaciones tal como lo expresa Giraldo [10].
Con el fin de exhibir las potencialidades del modelo de decisión, se plantean 4 escenarios que se muestran en la Tabla II. Las coordenadas $x, y$ son relativas al punto $(0,0)$ mostrado en la Fig. 3 y los valores de las demás variables de decisión se dan en las unidades de medida referenciados en la Tabla I. Los tiempos de molienda y lavado de caña se muestrean según una distribución de probabilidad normal (al ser posible utilizar otra distribución de probabilidad). Cabe destacar que la posibilidad de escenarios es tan grande como las que imagine el experimentador. Los resultados de la experimentación de estos 4 escenarios se pueden ver en la Fig. 6. Los datos en los que se basan los gráficos pueden ser consultados en el sitio http://modelogpoeniq.wordpress.com/.

La Fig. 6, el gráfico 1 muestra claramente que los escenarios 2 y 4 alcanzan el más alto nivel de producción de bioetanol, mientras que los escenarios 1 y 3 son los peores. El gráfico 2 muestra el comportamiento del nivel de producción de etanol a través del tiempo. Dada la robustez de los modelos de simulación discutida anteriormente, es posible que el usuario obtenga otras mediciones del desempeño del sistema. Así, por ejemplo, el gráfico 3 , muestra que los escenarios 2 y 4 son los que logran una mayor tasa de utilización de la zona de molienda y el gráfico 4 señala que en los escenarios 1 y 3 el transportador recorre mayores distancias. 
TABLA II.

ESCENARIOS DE SIMULACIÓN PLANTEADOS

\begin{tabular}{|c|c|c|c|c|c|c|c|c|}
\hline & \multicolumn{2}{|c|}{ Escenario 1} & \multicolumn{2}{|c|}{ Escenario 2} & \multicolumn{2}{|c|}{ Escenario 3} & \multicolumn{2}{|c|}{ Escenario 4} \\
\hline & $\mathrm{x}$ & $\mathrm{Y}$ & $\mathrm{x}$ & $\mathrm{Y}$ & $\mathrm{x}$ & $\mathrm{Y}$ & $\mathrm{x}$ & $y$ \\
\hline Almacenamiento caña & -70 & 0 & -46 & 0 & -70 & 0 & -46 & 0 \\
\hline Lavado caña & -54 & 0 & -45 & 2 & -54 & 0 & -45 & 2 \\
\hline Acopio bagazo & -34 & -20 & -44 & -2 & -34 & -20 & -44 & -2 \\
\hline Capacidad molienda & \multicolumn{2}{|c|}{1000} & \multicolumn{2}{|c|}{1000} & \multicolumn{2}{|c|}{1000} & \multicolumn{2}{|c|}{1000} \\
\hline Capacidad lavado de caña & \multicolumn{2}{|c|}{1000} & \multicolumn{2}{|c|}{1000} & \multicolumn{2}{|c|}{2000} & \multicolumn{2}{|c|}{2000} \\
\hline Tiempo molienda & \multicolumn{2}{|c|}{$\begin{array}{l}\text { Dist. normal } \\
\text { Media } 1 \mathrm{~h} \\
\text { Desv. } 0.1 \mathrm{~h}\end{array}$} & \multicolumn{2}{|c|}{$\begin{array}{l}\text { Dist. normal } \\
\text { Media } 1 \mathrm{~h} \\
\text { Desv. } 0.1 \mathrm{~h}\end{array}$} & \multicolumn{2}{|c|}{$\begin{array}{l}\text { Dist. normal } \\
\text { Media } 2 \mathrm{~h} \\
\text { Desv. } 0.1 \mathrm{~h}\end{array}$} & \multicolumn{2}{|c|}{$\begin{array}{l}\text { Dist. normal } \\
\text { Media } 2 \mathrm{~h} \\
\text { Desv. } 0.1 \mathrm{~h} \\
\end{array}$} \\
\hline Tiempo lavado de caña & \multicolumn{2}{|c|}{$\begin{array}{l}\text { Dist. normal } \\
\text { Media } 1 \mathrm{~h} \\
\text { Desv. } 0.1 \mathrm{~h}\end{array}$} & \multicolumn{2}{|c|}{$\begin{array}{l}\text { Dist. normal } \\
\text { Media } 1 \mathrm{~h} \\
\text { Desv. } 0.1 \mathrm{~h}\end{array}$} & \multicolumn{2}{|c|}{$\begin{array}{l}\text { Dist. normal } \\
\text { Media } 2 \mathrm{~h} \\
\text { Desv. } 0.1 \mathrm{~h}\end{array}$} & \multicolumn{2}{|c|}{$\begin{array}{l}\text { Dist. normal } \\
\text { Media } 2 \mathrm{~h} \\
\text { Desv. } 0.1 \mathrm{~h}\end{array}$} \\
\hline
\end{tabular}

Fig. 6. RESULTADOS DE LA SIMULACIÓN DE 4 ESCENARIOS A 5 RÉPLICAS

Gráfico 1

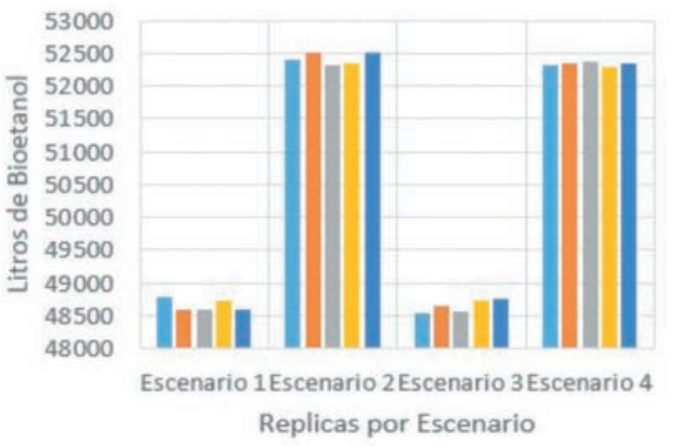

Gráfico 3

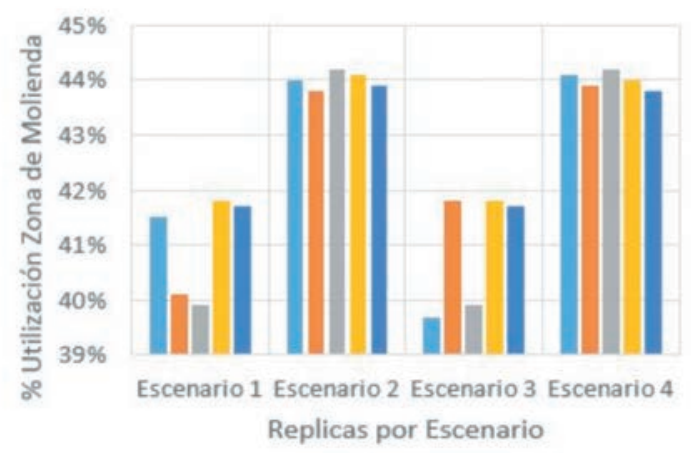

Gráfico 2

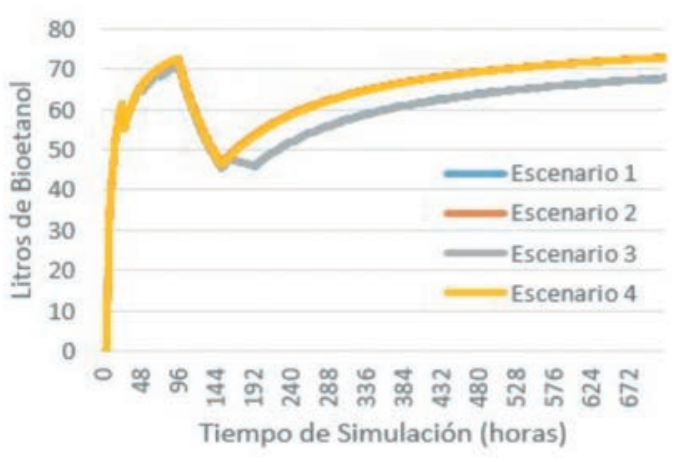

Gráfico 4

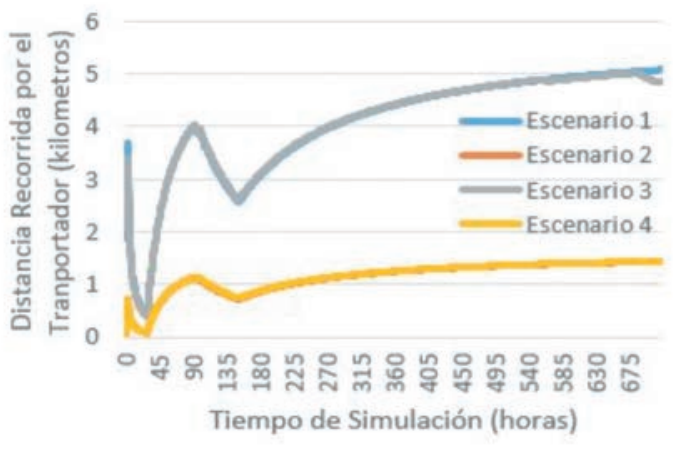

Fuente: autores.

En síntesis, los resultados de la simulación muestran cómo para los escenarios planteados las variables de decisión relacionadas con la distribución en planta tienen un gran efecto en la variable resultado, esto se corrobora con el gráfico 4 de la Fig. 6, en el cual los escenarios que pre- sentaron menor desplazamiento son los mismos escenarios donde la producción de bioetanol es mayor.

Con el fin de que el lector vea otros resultados y compruebe de nuevo la robustez del modelo, en el sitio http://modelogpoeniq.wordpress.com/ 
encontrará los resultados de simular otros 4 escenarios que miden el desempeño en términos de producción de bioetanol, tasa de utilización de la zona de lavado de caña y el transportador. Además, en el sitio referenciado, mediante el enlace Encuesta de Satisfacción, los estudiantes pueden calificar su experiencia en el uso del modelo en términos de: comprensión del modelo, aporte a su formación, cumplimiento de expectativas, entre otros.

\section{CONCLUSIONES}

La cualidad más importante del modelo desarrollado es la posibilidad de representar la alta variabilidad e interdependencia de los sistemas, como el de producción de bioetanol. Como se pudo comprobar, esta variabilidad se representó muestreando tiempos de proceso con distribuciones de probabilidad. La interdependencia se representó incluyendo del sistema real sus componentes principales y comportamiento entre estos.

El modelo de decisión desarrollado permite a los estudiantes de Ingeniería Química entrenarse para la toma de decisiones en temas relacionados con la GPO, dado que la simulación permite experimentar con una abstracción de un proceso real sin afectarlo ni correr con los riesgos y costos que esto conlleva.

Dado la robustez del modelo planteado, un estudio y análisis con otras herramientas, tales como la programación lineal (la cual supone que el comportamiento del mundo real es lineal), generaría resultados alejados de la realidad.

Igualmente, es posible con los resultados de producción de bioetanol efectuar análisis de varianza (ANOVA), a fin de determinar estadísticamente que variables de decisión tienen un impacto importante en el nivel de producción de bioetanol. Dado el alto volumen de escenarios posibles $\left(2^{10}\right)$, se sugiere emplear diseños fraccionados, según se considera en diseño experimental $2^{k}$.

\section{AGRADECIMIENTOS}

Los autores agradecen a la Universidad $\mathrm{Na}$ cional de Colombia Sede Manizales, a través de la Dirección de Investigaciones (DIMA) y al Departamento de Ingeniería Industrial por su apoyo institucional al desarrollo de esta investigación (proyecto: Modelo de soporte al proceso ense- ñanza-aprendizaje de la gestión de la producción y operaciones basado en TICS. Aplicación al programa de Ingeniería Química, Código Hermes: 21817) el cual hace parte de la tesis de Maestría del coautor Jhonathan M. Vargas.

\section{REFERENCIAS}

[1] A. M. Sánchez Riaño, A. I. Gutiérrez Morales, J. A. Muñoz Hernández, y C. A. Rivera Barrero, "Producción de bioetanol a partir de subproductos agroindustriales lignocelulósicos". Tumbaga, pp. 61-91, 2010.

[2] M. T. Hernández Nodarse, "Tendencias actuales en la producción de bioetanol", Ingeniería Primero, pp. 1-17, 2008.

[3] H. J. Vázquez, y O. Dacosta, “Fermentación alcohólica: una opción para la producción de energía renovable a partir de desechos agrícolas", Ingeniería Investigación y Tecnología, pp. 249-259. 2007.

[4] C. J. Geankoplis, "Procesos de transporte y operaciones unitarias", México D. F.: Compañía Editorial Continental, S.A. DE C.V., 1998.

[5] D. M. Cárdenas Aguirre, Ó. D. Castrillón Gómez, F. Becerra Rodríguez, A. García, J. A. Giraldo García, S. Ibarra Mirón, A. Zapata Gómez, "Gestión de la Producción: Una Aproximación Conceptual”, Bogotá: Universidad Nacional de Colombia, Unibiblos, 2008.

[6] Congreso de la República de Colombia. "Ley 18 de 1976. Por la cual se reglamenta el ejercicio de la profesión de Ingeniero Químico en el país, reconocida por el Ministerio de Educación Nacional”, Bogotá. 1976.

[7] W. D. Kelton y A. M. Law, "Simulation Modeling and Analysis 3nd Ed”, McGraw-Hill. 2007.

[8] J. Banks, J. S. Carson, B. L. Nelson, "Discrete-Event System Simulation, 4a Ed.”, Nueva York: Prentice Hall, 2009.

[9] E. R. Shannon, “Simulación de Sistemas, 1a ed.", México: Editorial Trillas, 1988.

[10] J. A Giraldo García, "Simulación de sistemas de producción y de servicios", Universidad Nacional de Colombia, Facultad de Ingeniería y Arquitectura, 2014.

[11] C. A. Cardona, O. J. Sánchez, M. I. Montoya y J. A. Quintero, "Simulación de los procesos de obtención de etanol a partir de caña de azúcar y maíz", Revista Scientia et Technica, pp. 187-192, 2005.

[12] P. J. Camacho, J. J. Martínez, C. A. Cardona y O. J. Sánchez, "Simulación del proceso de producción de bioetanol a partir de biomasa residual de microalga obtenida como subproducto de la producción de bio- 
diesel de tercera generación" Trabajo de Grado para obtener el título de Ingeniero Químico, Universidad Industrial de Santander, 2011.

[13] G. Gonzáles, "Modelado y simulación de una planta de producción de etanol por Hidratación Directa de Etileno", Trabajo de Grado para obtener el título de ingeniero Químico, Universidad de Sevilla, 2011.

[14] J. M. Vargas, "Proyecto de Tesis de Maestría: Modelo de soporte al proceso enseñanza/aprendizaje de la gestión de la producción y operaciones basado en TIC. Aplicación al Programa de Ingeniería Química”, Universidad Nacional de Colombia sede Manizales, 2013.
[15] C. A. Fredes, J. P. Hernández, y D. A. Díaz, “Potencial y problemas de la simulación en ambientes virtuales para el aprendizaje", Formación Universitaria, pp. 4556. 2012

[16] C. A. López, y C. A. Saiegg, “Uso de la simulación como estrategia de mejora en el proceso de enseñanzaaprendizaje en las universidades. Una Aplicación para la carrera de Informática. Tesis de Licenciatura en Informática", Comodoro Rivadavia: Universidad Nacional de la Patagonia San Juan Bosco. 2005. 